\title{
Factors associated with benign multiple sclerosis in the New York State MS Consortium (NYSMSC)
}

\author{
Robert Zivadinov ${ }^{1,2^{*}}$, Diane L. Cookfair ${ }^{2}$, Lauren Krupp ${ }^{3}$, Aaron E. Miller ${ }^{4}$, Neil Lava ${ }^{5}$, Patricia K. Coyle ${ }^{3}$, \\ Andrew D. Goodman ${ }^{6}$, Burk Jubelt ${ }^{7}$, Michael Lenihan ${ }^{8}$, Joseph Herbert ${ }^{9}$, Malcolm Gottesman ${ }^{10}$, \\ David H. Snyder ${ }^{11,12}$, Brian R. Apatoff ${ }^{13}$, Barbara E. Teter ${ }^{2}$, Allan B. Perel ${ }^{14}$, Frederick Munschauer $^{2}$ \\ and Bianca Weinstock-Guttman²
}

\begin{abstract}
Background: This retrospective analysis explored prognostic factors associated with a benign multiple sclerosis (BMS) disease course at baseline and over the 4-year follow-up.

Methods: Patients from the centralized New York State Multiple Sclerosis Consortium registry were classified as having BMS according to 3 different criteria centered on disease duration and disability. Additional analyses explored prognostic factors associated with BMS using the most conservative disability criteria (Expanded Disability Status Scale $\leq 2$ and disease duration $\geq 10$ years).

Results: Among 6258 patients who fulfilled eligibility criteria, $19.8 \%$ to $33.3 \%$ were characterized as having BMS, at baseline depending on classification criteria used. Positive prognostic factors for BMS at baseline included female sex ( $p<0.0001$ ) and younger age at onset $(p<0.0001)$; negative prognostic factors included progressive-onset type of MS and African-American race. Of the 1237 BMS patients (per most conservative criteria), 742 were followed for a median of 4 years to explore effect of disease-modifying treatment (DMT) on benign status. DMT $(p=0.009)$ and longer disease duration ( $p=0.007$ ) were the only significant positive predictors of maintaining BMS at follow-up. The protective effect was stronger for patients taking DMT at both enrollment and follow-up ( $\mathrm{OR}=0.71 ; p=0.006)$.
\end{abstract}

Conclusions: There is a need for development of more reliable prognostic indicators of BMS. Use of DMT was significantly associated with maintaining a benign disease state.

Keywords: Benign multiple sclerosis, Disease course, Disease-modifying treatment, DMT, Multiple sclerosis

\section{Background}

Multiple sclerosis (MS) is an immune-mediated neurodegenerative disorder of the central nervous system with a lifetime risk of 1 in 400 in Caucasians in industrialized society [1]. The disease course is highly heterogeneous and includes a subgroup of patients with benign MS (BMS) who demonstrate little disease progression and minimal disability, even decades after clinical onset [2-5]. However,

\footnotetext{
* Correspondence: rzivadinov@bnac.net

Deceased

${ }^{1}$ Buffalo Neuroimaging Analysis Center, Department of Neurology, School of Medicine and Biomedical Sciences, University at Buffalo, Buffalo, NY, USA ${ }^{2}$ Department of Neurology, School of Medicine and Biomedical Sciences, University at Buffalo, 100 High Street, Buffalo, NY 14203, USA

Full list of author information is available at the end of the article
}

there is no universally agreed upon definition of BMS, as multiple patient profiles are associated with this disease course [2].

In 1996, an international survey conducted by the National Multiple Sclerosis Society (NMSS) defined BMS as a disease in which patients remain fully functional across neurologic systems 15 years after disease onset $[6,7]$. Unfortunately, the term "fully functional" was not clearly defined, resulting in considerable variations in the estimated frequency of BMS [8-10].

Benign MS is a retrospective diagnosis that can only be made 10 years or more after disease onset. Despite consensus recommendations for immediate treatment following diagnosis, there is still a difference of opinion among 
physicians regarding this issue because of the paucity of efficacy data with disease modifying therapies (DMTs) in patients with BMS [11-15].

The New York State MS Consortium (NYSMSC) has assembled a centralized registry of demographic and clinical information from more than 7000 patients with clinically definite MS from across New York State (NYS), providing a robust dataset to study the frequency and characteristics of BMS. [16, 17] This study aimed to (a) retrospectively estimate the prevalence of BMS based on the NYSMSC registry using the 3 most commonly used BMS criteria [2, 3, 18]; (b) evaluate prognostic factors associated with BMS; (c) investigate the course of BMS prospectively over time $[2,8,19]$; and (d) explore whether DMT use correlated with maintaining a benign status in patients classified as having BMS at time of study enrollment.

\section{Methods Study details}

This was a retrospective cohort study characterized by demographic, clinical, and treatment information available from the NYSMSC centralized registry. The NYSMSC is a regional affinity group of $18 \mathrm{MS}$ centers throughout NYS established to promote MS research and enhance patient care $[16,20]$. This study consisted of 2 parts: a crosssectional comparison of patients at the time of enrollment in the registry (i.e. baseline evaluation) and the assessment of patients with follow-up data. Patient enrollment in the NYSMSC registry began on February 14, 1996. This date was also the start of study enrollment. All patients with a clinically definite MS diagnosis (CDMS) according to the Poser criteria [21] enrolled in the NYMSC registry from the start of the registry until December 31, 2002, were included in the study cohort. While this later date represented the final enrollment date for inclusion in the cohort (i.e. December 31, 2002), a follow-up cut-off date of October 1, 2004, was imposed for patient follow-up data. This follow-up cut-off date was chosen to provide similar diagnosis criteria (CDMS), at least 1 year of follow-up for the most recently enrolled patients, and because it represented approximately 10 years since the first DMT approved for treatment of MS became available. A complete baseline evaluation at NYSMSC enrollment, including completion of the demographic and clinical sections of the NYSMSC standardized data collection instrument, was also required for study inclusion.

The standardized data collection instrument consists of 2 sections: Section 1 contains demographic data and self-report assessments that are completed by patients during their regularly scheduled neurologic appointment; Section 2, completed by the examining neurologist and/or nurse practitioner certified in Expanded Disability Status Scale (EDSS) performance, includes signs and symptoms at onset, relapse history, MS type, EDSS score, and use of DMTs. All clinical, demographic, and treatment data from enrollment and yearly ( \pm 6 months) follow-up visits from all participating NYSMSC sites are maintained at a centralized data management center (UDSMR) [16, 17].

Patients were defined as receiving DMT, if they received any of the following Food and Drug Administration (FDA)-approved treatments and/or investigational DMTs: FDA-approved DMTs-interferon beta-1a/b (IFN- $\beta 1 \mathrm{a} / \mathrm{b}$; Avonex $^{\circ}$, Rebif ${ }^{\oplus}$, Betaseron $\left.{ }^{\circ}\right)$, glatiramer acetate (Copaxone $^{\circ}$ ), mitoxantrone (Novantrone ${ }^{\odot}$ ), and natalizumab (Tysabri ${ }^{\circ}$ ); investigational DMTs (taken by $\sim 1 \%$ of patients) - cyclophosphamide, intravenous immunoglobulin, oral myelin, linomide, and azathioprine. All patients receiving treatments that were not DMTs (e.g. symptomatic medications) were included in the not-treated group for the various time points unless they also received a DMT.

The study was approved by the relevant Institutional Review Boards (IRBs) of the participating centers and written informed consent was obtained by all study participants prior to enrollment in the NYSMSC registry. The IRB renewals were obtained annually on an ongoing basis throughout the study. The permission was obtained for using the NYSMSC database by the NYSMSC Scientific Committee, which approved the study protocol.

\section{Patient classification}

Patients were retrospectively classified as having BMS or non-BMS at baseline (i.e. NYSMSC enrollment) according to the 3 most commonly used benign criteria: EDSS $\leq 2$ and disease duration (DD) $\geq 10$ years (Criterion I), EDSS $\leq 3$ and DD $\geq 15$ years (Criterion II), and EDSS $\leq 3$ and DD $\geq 10$ years (Criterion III) (Table 1) [2, 3, 18]. Disease duration was defined as time from first symptom onset. Prevalence of BMS at enrollment according to these 3 classifications was evaluated. Additional baseline and follow-up analyses were carried out for the first benign classification criteria (EDSS $\leq 2$ and DD $\geq 10$ years) because this classification was the most conservative with respect to EDSS (referred to herein as Criterion I). Within the context of this classification, patients were placed into 1 of 2 categories: BMS (i.e. EDSS $\leq 2$ and $\mathrm{DD}<10$ years) and non-BMS (i.e. EDSS $\leq 2$ and DD $<10$; years or EDSS $>2$ regardless of DD). For this latter category, the worsening to EDSS $>2$ could have occurred at any time during the disease course.

\section{Statistical methods}

All statistical analyses were performed using Statistical Package for Social Sciences (SPSS Incorporated, Chicago, IL, version 22.0). The association between clinical and demographic variables for Criterion I benign, vs. non- 
Table 1 Baseline demographic and clinical characteristics of patients with benign MS based on 3 classification criteria

\begin{tabular}{|c|c|c|c|}
\hline Variable & $\begin{array}{l}\text { EDSS } \leq 2, \mathrm{DD} \geq 10^{\mathrm{a}} \\
\text { (Criterion I) }\end{array}$ & $\begin{array}{l}\mathrm{EDSS} \leq 3, \mathrm{DD} \geq 15 \\
\text { (Criterion II) }\end{array}$ & $\begin{array}{l}\text { EDSS } \leq 3, \mathrm{DD} \geq 10 \\
\text { (Criterion III) }\end{array}$ \\
\hline Patients, n (\%) & $1237(19.8)$ & $1253(20.0)$ & 2081 (33.3) \\
\hline Female, \% & 79.6 & 78.5 & 77.4 \\
\hline $\begin{array}{l}\text { Race, } \% \\
\text { Caucasian/Hispanic-American } \\
\text { African-American } \\
\text { Other }\end{array}$ & $\begin{array}{l}94.6 \\
3.8 \\
1.6\end{array}$ & $\begin{array}{l}95.3 \\
3.6 \\
1.1\end{array}$ & $\begin{array}{l}94.2 \\
4.3 \\
1.3\end{array}$ \\
\hline Family history,\% & 19.5 & 20.6 & 20.2 \\
\hline Age at onset, y, mean (SD) & $30.2(8.5)$ & $29.3(8.6)$ & $30.8(8.9)$ \\
\hline Age at baseline, $y$, mean (SD) & $47.8(9)$ & $51.9(9)$ & $49.3(9.5)$ \\
\hline EDSS, mean (SD) & $1.4(0.6)$ & $2.1(0.8)$ & $2.0(0.8)$ \\
\hline EDSS, median (range) & $1.5(0-2.0)$ & $2.0(0-3.0)$ & $2.0(0-3.0)$ \\
\hline Disease duration, y, mean (SD) & $17(6.7)$ & $22(6.9)$ & $17.9(7.3)$ \\
\hline $\begin{array}{l}\text { Disease course, \% } \\
\text { Relapsing-remitting } \\
\text { Secondary-progressive } \\
\text { Progressive-relapsing } \\
\text { Primary-progressive }\end{array}$ & $\begin{array}{l}95.6 \\
2.7 \\
0.6 \\
1.1\end{array}$ & $\begin{array}{l}86.4 \\
9.3 \\
1.8 \\
2.5\end{array}$ & $\begin{array}{l}88.4 \\
7.5 \\
1.5 \\
2.6\end{array}$ \\
\hline On DMT at enrollment, $\mathrm{n}(\%)^{\mathrm{c}}$ & $505(40.8)$ & $481(38.4)$ & $848(40.7)$ \\
\hline $\begin{array}{l}\text { Type of DMT, n (\%) } \\
\text { Interferon beta-1a IM } \\
\text { Interferon beta-1b SC } \\
\text { Interferon beta-1a SC } \\
\text { Glatiramer acetate } \\
\text { Methotrexate } \\
\text { Myelin (oral) } \\
\text { Mitoxantrone } \\
\text { Azathioprine } \\
\text { Cyclophosphamide } \\
\text { Immunoglobulin (intravenous) } \\
\text { Linomide }\end{array}$ & $\begin{array}{l}314(62.2) \\
98(19.4) \\
1(0.2) \\
64(12.7) \\
8(1.6) \\
13(2.6) \\
0 \\
3(0.6) \\
1(0.2) \\
2(0.4) \\
1(0.2)\end{array}$ & $\begin{array}{l}300(62.4) \\
90(18.7) \\
1(0.2) \\
59(12.3) \\
12(2.5) \\
11(2.3) \\
0 \\
5(1.0) \\
1(0.2) \\
1(0.2) \\
1(0.2)\end{array}$ & $\begin{array}{l}515(60.7) \\
164(19.3) \\
3(0.4) \\
107(12.6) \\
19(2.2) \\
24(2.8) \\
0 \\
7(0.8) \\
3(0.4) \\
4(0.5) \\
2(0.2)\end{array}$ \\
\hline
\end{tabular}

$D D$ disease duration (years from symptom onset to enrollment)

Abbreviations: DMT disease-modifying therapy, EDSS Expanded Disability Status Scale, MS multiple sclerosis

${ }^{a}$ Most conservative definition

${ }^{\mathrm{b}}$ Out of $n=6258$ total cohort patients

'Were being treated with disease modifying therapy (DMT) at time of enrollment in the NYSMSC registry

BMS patients was first assessed using chi-square, $t$-test or Mann-Whitney $u$ test, as appropriate.

Odds ratios and $95 \%$ confidence intervals for predictors of benign vs. non-BMS status adjusted for other predictors at baseline were calculated using binary logistic regression (LR). For these comparisons, non-BMS patients were limited to those with $\geq 10$ years DD so as to make the two groups similar with regard to minimum disease duration. Cox regression models were used to explore the effect of DMT use from enrollment through follow-up on benign status over time while simultaneously adjusting for significant baseline predictors of benign status in a subset of Criterion I benign patients with follow-up data $(n=742)$. Hazard ratios (HRs) and $95 \%$ confidence intervals were calculated from final Cox models. Backward stepwise modeling was used for both LR and Cox regression, with entry criteria set at $p<1.00$ and removal criteria at $p<0.05$. Additional details regarding regression models are provided in the footnotes of Table 3.
All models were initially run with a full set of variables identified as predictors during univariate analyses, with one exception. Because age-related variables were highly correlated, some were eliminated from the model to ensure that final fitted models met the underlying assumptions of multicollinearity and Homer-Lemshow goodness-of-fit criteria (LR). Detailed information regarding variables included in the models are provided in the footnotes for Table 3 and Fig. 1.

Data regarding DMTs taken by individual Criterion I patients included type of DMT taken at time of enrollment and follow-up. Data regarding specific duration of DMT use were not available. Therefore, 2 analyses were conducted. In the first, DMT use was classified as Ever ( $n=$ $633)$ or Never $(n=109)$ used as of the end of follow-up. For the second analysis, 4 categories were established using all DMT data as of follow-up: (1) Never used DMT ( $n=109)$, (2) Was using DMT at time of enrollment but not at follow-up ( $n=83)$, (3) Used DMT at time of follow- 

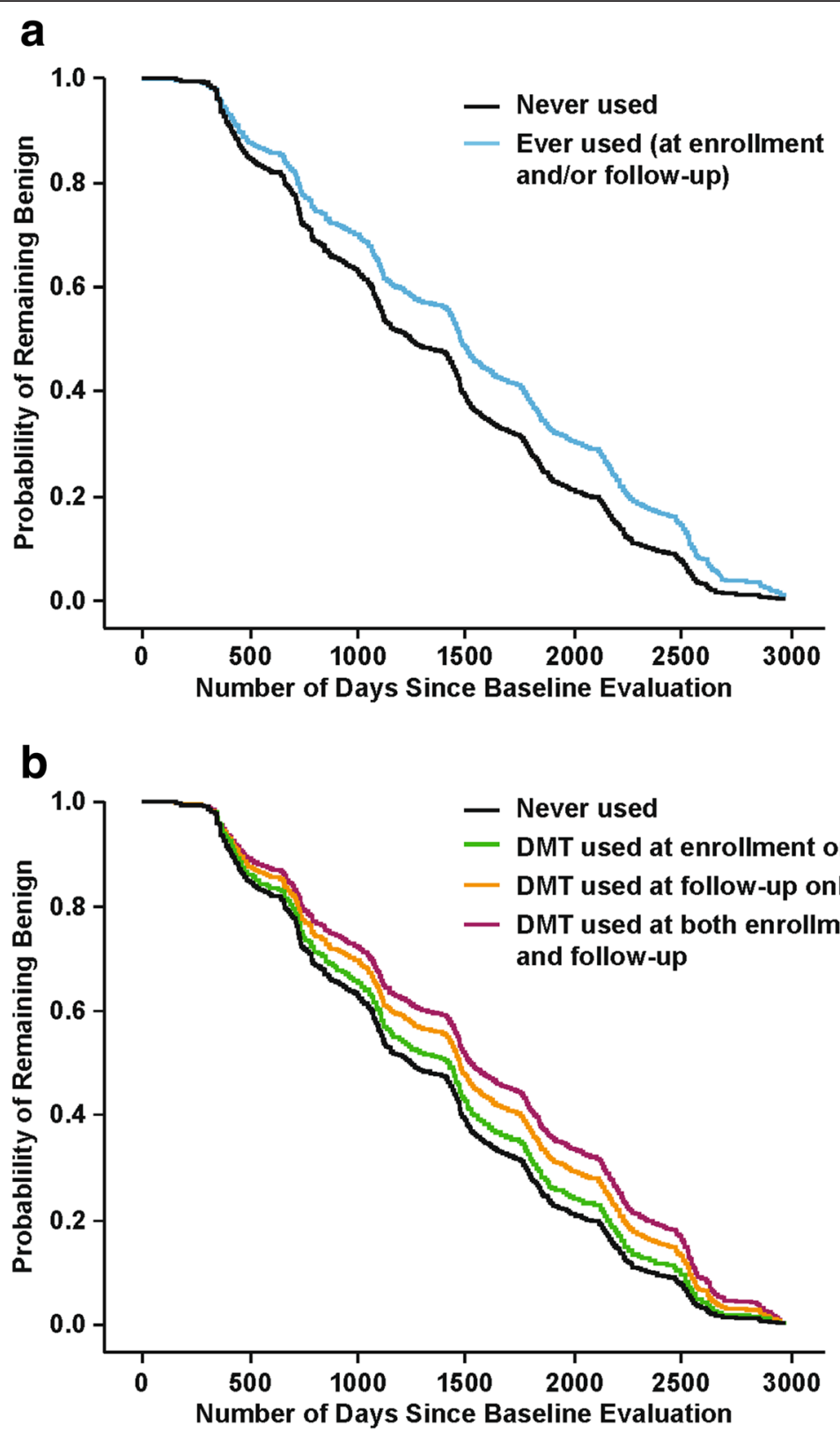

Fig. 1 Cox regression survival curves. Probability of remaining benign for $n=742$ Criterion I benign MS patients for (a) 2-category definition of DMT use: Ever ( $n=633$ ) vs Never ( $n=109 ; p=0.009)$ and (b) 4-category definition of DMT use: Never Used ( $n=109$, comparison group); at enrollment only ( $n=83$, not significant); at follow-up only ( $n=258, p=0.038)$; at both enrollment and follow-up $(n=292, p=0.006)$. Abbreviations: DMT, disease-modifying therapy. Data from New York State Multiple Sclerosis Consortium

up but not on DMT at enrollment $(n=258)$, and (4) Was on DMT at enrollment and also at follow-up $(n=292)$. For this second analysis, the Never used DMT group served as the reference category for the other 3 groups. Forty-two benign patients from the Buffalo, NY, site who were not on treatment at enrollment or follow-up had a history of IFN- $\beta 1$ a use before its approval in 1996 as a result of participation in the IFN- $\beta 1$ a phase 3 trial. These patients $(n=42)$ were excluded from the Cox model analyses exploring the effect of DMTs on benign status. Four additional patients were excluded from Cox model analyses because of missing data on DMT use at enrollment.

\section{Results}

Demographic and clinical characteristics at baseline Of the 7158 patients enrolled in the NYSMSC registry, 6258 patients fulfilled the inclusion criteria and were 
included in the study (Additional file 1: Figure S1). The majority (61.9\%) of patients in the study cohort had relapsing-remitting (RRMS), while approximately one quarter of patients $(26.5 \%)$ had secondary-progressive MS (SPMS). Only $8.0 \%$ of patients had primary-progressive MS (PPMS), and $<4 \%$ had progressive-relapsing (PR) MS (Additional file 1: Figure S1). Disease course was determined per NMSS recommended criteria available at the time of study enrollment [6].

At baseline, $19.8 \%(n=1237)$ of patients in the total study cohort were classified as having BMS according to the most conservative classification criteria (Criterion I; Table 1). Similarly, $20 \%(n=1253)$ of patients were classified as having BMS using Criterion II. In contrast, when using the least conservative classification (Criterion III), $33.3 \%(n=2081)$ of patients were classified as having BMS (Table 1).

The descriptive data in Table 1 suggest that some demographic and clinical characteristics differed according to criteria, especially for Criterion I vs Criterion II. Certain differences were expected based on criteria definitions (e.g. lower mean EDSS for benign patients in the Criterion I group vs those in Criterion II and III groups, and shorter mean disease durations for BMS in the Criterion I [17 years] and III groups [17.9 years] compared with those in the Criterion II group [22 years]). Disease course also varied for patients defined as having BMS based on Criterion I vs Criterion II and III (Table 1). The percentage of patients with RRMS was higher among Criterion I BMS patients $(95.6 \%)$ than among Criterion II (86.4 \%) and Criterion III (88.4\%) BMS patients.

In the total cohort of MS patients $(n=6258)$, use of the most conservative classification criteria (Criterion I) resulted in $19.8 \%$ of patients classified as benign at baseline. $80.2 \%$ of patients were classified as having non-BMS (Table 2). In univariate analyses comparing Criterion I BMS patients with non-BMS patients at baseline, significant differences between patients with BMS, vs non-BMS patients were seen with respect to nearly all baseline demographic and clinical characteristics assessed with the exception of family history of MS (Table 2). Less than $1 \%$ of the total cohort who met Criteria I criteria for benign status had progressive disease (SP, PP or SP). Removing these patients from the analysis made no difference in statistical significance of results, nor did considering them non-BMS due to their status as progressive patients (Table 2). Positive predictors of conservative-criteria BMS at baseline included female sex $(p<0.0001)$ and younger age at onset $(p<0.0001)$. Patients with progressive-onset type of MS and those of African-American race were less likely to have BMS at baseline (Table 2). Of the 382 African-American patients included in the study cohort, $11.8 \%$ were classified as having Criterion I BMS. In LR models, comparing benign (EDSS $\leq 2$ ) with non-
BMS patients with $\mathrm{DD} \geq 10$ years for both groups, findings were similar to those of univariate 2 -group comparisons, except that BMS patients were more likely to have a later age at onset than non-BMS patients after adjusting for other variables in the model.

\section{Characteristics of criterion I benign patients at follow-up} Follow-up data were available for significantly fewer patients $(n=3583,57.3 \%)$ compared with the original cohort (median cohort follow-up 3 years, range 1-8 years). Of the 1237 (19.8\%) patients originally classified as having BMS at baseline per Criterion I, 788 (63.7 \%) had followup data available (median follow-up 4 years, range 1-8 years). Of these patients, $64.8 \%(n=511)$ continued to present with BMS at follow-up, whereas the remaining $35.2 \%$ of patients $(n=277)$ had progressed to non-BMS.

\section{Factors associated with loss to follow-up}

Logistic regression analyses also were used to evaluate factors predicting loss to follow-up. The full model tested included sex, race, disease course, DD, age at disease onset, and Criterion I classification (i.e. benign vs nonBMS). Variables that predicted higher loss to follow-up included having SPMS $(p<0.0001)$ or PPMS $(p<0.05)$ course; longer DD $(p<0.05)$; and being in the non-BMS group $(p<0.0001)$.

\section{DMTs in benign patients by criterion I cohort at baseline and follow-up}

Most patients on DMT at enrollment and/or follow-up were on 1 of 3 DMTs: (1) IFN- $\beta 1$ a (Avonex) - enrollment only, $n=33$; follow-up only, $n=157$; (2) IFN- $\beta 1 \mathrm{~b}$ (Betaseron)-enrollment only, $n=27$; follow-up only, $n=37$; or (3) glatiramer acetate (Copaxone)-enrollment only, $n=9$; follow-up only, $n=53$. Eight patients were taking methotrexate alone at enrollment, and 1 at follow-up. One patient was taking subcutaneous IFN- $\beta 1$ 1a (Rebif) at enrollment, and 6 at follow-up. Other DMTs taken by $\leq 1.2 \%$ of patients at enrollment or follow-up were cyclophosphamide, mitoxantrone (Novantrone), intravenous immunoglobulin, oral myelin, linomide, and azathioprine.

Two-hundred ninety-two patients were on DMT at both enrollment and follow-up, including 173 on IFN- $\beta 1$ a (Avonex), 32 on glatiramer acetate (Copaxone), 44 on IFN- $\beta 1 b$ (Betaseron), and 1 on IFN- $\beta 1$ a (Rebif). The remainder of patients on DMT at both time points $(n=42)$ changed DMT between enrollment and follow-up. One hundred nine patients (14.7 \%) had no history of DMT use at enrollment and did not use DMT during the study.

\section{Effect of DMT on continued benign status in criterion I cohort}

Cox models were used to simultaneously evaluate significant baseline predictors of benign status and DMT 
Table 2 Baseline demographic and clinical characteristics of MS patients with benign MS according to classification Criterion I (EDSS $\leq 2$ and DD $\geq 10$ years) vs. non-benign MS patients

\begin{tabular}{|c|c|c|c|}
\hline Variable & BMS (EDSS $\leq 2, \mathrm{DD} \geq 10)^{\mathrm{a}}$ & Non-BMS & $p$-value ${ }^{*}$ \\
\hline All Patients, n (\%) ${ }^{\mathrm{b}}$ & $1237(19.8)$ & $5021(80.2)$ & $<0.0001$ \\
\hline Female, n (\%) & 985 (79.6) & $3590(71.4)$ & $<0.0001$ \\
\hline $\begin{array}{l}\text { Race, } \mathrm{n},(\%) \\
\text { Caucasian/Hispanic-American } \\
\text { African-American } \\
\text { Other }\end{array}$ & $\begin{array}{l}1170(94.6) \\
47(3.8) \\
20(1.6)\end{array}$ & $\begin{array}{l}4617(91.9) \\
329(6.6) \\
75(1.5)\end{array}$ & $<0.0001$ \\
\hline Family history, n, (\%) & $241(19.5)$ & $976(19.4)$ & NS \\
\hline Age at onset, y, mean (SD) & $30.2(8.5)$ & $32.2(9.7)$ & $<0.0001$ \\
\hline Age at baseline, $y$, mean (SD) & $47.8(9.0)$ & $51.8(10.5)$ & $<0.0001$ \\
\hline EDSS, mean (SD) & $1.4(0.6)$ & $4.4(1.6)$ & $<0.0001$ \\
\hline EDSS, median (range) & $1.5(0-2.0)$ & $5.0(0-9.5)$ & $<0.0001$ \\
\hline Disease duration, $y$, mean $(\mathrm{SD})^{c}$ & $17.0(6.7)$ & $18.3(7.5)$ & $<0.0001$ \\
\hline $\begin{array}{l}\text { Disease course,(n)\% }{ }^{\mathrm{d}} \\
\text { Relapsing-remitting } \\
\text { Secondary-progressive } \\
\text { Progressive-relapsing } \\
\text { Primary-progressive }\end{array}$ & $\begin{array}{l}1184(95.6) \\
33(2.7) \\
7(0.6) \\
13(1.1)\end{array}$ & $\begin{array}{l}2692(53.6) \\
1622(32.3) \\
219(4.4) \\
488(9.7)\end{array}$ & $<0.0001$ \\
\hline DMT at enrollment, $\mathrm{n}(\%)^{\mathrm{e}}$ & $505(40.8)$ & $2182(81.2)$ & $p<0.0001$ \\
\hline $\begin{array}{l}\text { Type of DMT, n (\%) } \\
\text { Interferon beta-1a IM } \\
\text { Interferon beta-1b SC } \\
\text { Interferon beta-1a SC } \\
\text { Glatiramer acetate } \\
\text { Methotrexate } \\
\text { Myelin (oral) } \\
\text { Mitoxantrone } \\
\text { Azathioprine } \\
\text { Cyclophosphamide } \\
\text { Immunoglobulin } \\
\text { (intravenous) } \\
\text { Linomide } \\
\text { T-cell vaccine }\end{array}$ & $\begin{array}{l}314(62.2) \\
98(19.4) \\
1(0.2) \\
64(12.7) \\
8(1.6) \\
13(2.6) \\
0(0.0) \\
3(0.6) \\
1(0.2) \\
2(0.4) \\
1(0.2) \\
0(0.0)\end{array}$ & $\begin{array}{l}1063(48.7) \\
466(21.4) \\
16(0.7) \\
306(14.0) \\
206(9.4) \\
19(0.9) \\
20(0.9) \\
39(1.8) \\
23(1.1) \\
4(0.2) \\
19(0.8) \\
1(<0.1)\end{array}$ & \\
\hline
\end{tabular}

Abbreviations: MS multiple sclerosis, BMS benign MS, DMT disease-modifying therapy, EDSS Expanded Disability Status Scale, IM intramuscular, NS not significant, SC subcutaneous, $S D$ standard deviation

*Between-group comparisons were performed using chi-square, t-tests, or Mann-Whitney $U$ tests as appropriate

${ }^{a}$ Most conservative classification criteria definition

${ }^{\mathrm{b}}$ Denominators: Benign MS ( $\left.n=1237\right)$; Other MS $(n=5021)$

${ }^{\mathrm{C}} \mathrm{DD}$, disease duration (years from symptom onset to enrollment)

${ }^{d}$ At time of baseline enrollment, only 53 progressive patients (SP, PR, and PP) out of the entire cohort of $6,258(<1 \%)$ met classification Criterion la (EDSS $\leq 2$ and

$\mathrm{DD} \geq 10$ years) for benign MS. Removing these patients from the analysis made no difference in statistical significance of results, nor did considering them

non-benign due to their status as progressive patients

${ }^{e}$ DMT at enrollment, $n=2687$ (42.9\%) of total cohort ( $n=6258$ ) (benign MS on DMT, $n=505$; other MS on DMT $n=2182$ )

use in $n=742$ Criterion I baseline benign patients. The only significant predictors of remaining benign at follow-up in the final fitted model were Ever used DMT $(p=0.009)$ and longer DD $(p=0.007)$, both of which were protective (Table 3, Fig. 1a). Longer DD and DMT use were also the only variables remaining in the final model for the analysis in which DMTs were grouped into 4 categories. In this latter analysis, likelihood of remaining benign at follow-up for those who took DMT at time of enrollment but stopped taking DMT before follow-up was not significantly different from Never used DMT. However, a protective effect was seen for those patients taking DMT at followup $(\mathrm{HR}=0.80 ; p=0.037)$. Furthermore, the protective effect was stronger and more significant for patients taking DMT at both enrollment and follow-up ( $\mathrm{HR}=0.71 ; p=0.006)$ (Table 3 and Fig. 1b).

\section{Discussion}

Although various definitions for the classification have been proposed, BMS is generally characterized by the lack of disease progression and minimal disability 10 years or more after disease onset $[2,19,22]$. The considerable variation in the estimated frequency of BMS has the potential to influence treatment decisions because, historically, some clinicians were reluctant to prescribe DMTs for patients with little evidence of physical disability [10]. Our own data supports this observation. At time of enrollment into the study cohort, only $40.8 \%$ of benign MS patients 
Table 3 DMT use as a predictor of continued benign status at follow-up in those who were benign at enrollment $(n=742)^{a, b, c, d}$ (A) DMT use Ever $(n=633) /$ Never $(n=109)^{\text {e,f }}$

$\begin{array}{ll}\text { HR }(95 \% \mathrm{Cl}) & p \text { value } \\ 0.60(0.42-0.85) & 0.009 \\ 0.97(0.97-0.99) & 0.007\end{array}$

DMT use at enrollment and/or follow-up Disease duration (years) at enrollment $0.97(0.97-0.99)$

0.007

(B) DMT use 4-category Cox model ${ }^{\mathrm{e}, \mathrm{f}}$

HR $(95 \% \mathrm{Cl}) \quad p$ value

DMT use at enrollment only $(n=83)$

DMT use at follow-up only $(n=258)$

DMT use at both enrollment and

$0.97(0.74-2.38)$

NS

$0.80(0.64-0.99) \quad 0.037$

$0.71(0.57-0.88) \quad 0.006$

follow-up $(n=292)$

Disease duration (years) at enrollment

$C$ confidence interval, DMT disease-modifying therapy, $H R$ hazard ratio MS multiple sclerosis

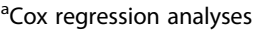

${ }^{\text {b}}$ The following variables were entered into the full Cox models comparing late benign with benign patients: sex, disease course, race, treatment status, age at onset and disease duration

${ }^{c}$ Continuous variables: age at onset (years), disease duration (years since symptom onset)

${ }^{d}$ Categorical variables: sex: female compared with male; disease course: secondary-progressive, progressive-relapsing, primary-progressive, compared with relapsing-remitting MS; race: African-American, Other, compared with Caucasian; treatment categories: See footnote $\mathrm{f}$, below

${ }^{e}$ Forty-six of 788 Criteria I baseline benign follow-up patients were excluded from Cox regression analyses, leaving a total of $N=742$ patients; $N=42$ patients who were not on treatment at enrollment or follow-up but had a history of IFN- $\beta 1$ a use prior to its approval in 1996 as a result of participation in the IFN- $\beta 1$ a phase 3 trial, and $N=4$ patients who were missing DMT data Never Users: $n=109$ patients had no history of DMT use at enrollment and did not use DMT at any time during the study. This group served as the reference category for DMT use in the Ever/Never and 4-category Cox models. DMTs used included interferon beta-1a/b (Avonex ${ }^{\oplus}$, Rebif ${ }^{\oplus}$, Betaseron $\left.{ }^{\oplus}\right)$, glatiramer acetate (Copaxone ${ }^{\circledast}$, azathioprine, cyclophosphamide, intravenous immunoglobulin methotrexate, linomide, myelin therapy; mitoxantrone, and natalizumab (1 patient on clinical trial)

'There was no difference in likelihood of remaining benign vs converting to late non-benign during follow-up according to sex, disease course, race, or age at onset after adjusting for significant predictors included in the final model

were on DMTs, whereas 81.8 \% of non-BMS patients (more than twice the rate of BMS patients, $p<0.0001$ ) were on DMTs (Table 2). Current recommendations propose DMTs for patients with MS from date of diagnosis without waiting to determine whether they meet the criteria for benign disease $[11,13,23]$.

This large study, which included 6258 patients enrolled in the NYSMSC registry, evaluated the frequency and characteristics of BMS in a prevalence cohort including patients from 18 centers across NYS accrued over a 6-year time period. Patients receiving investigational DMTs were included in the study to maintain the completeness of the dataset. The large sample size of this prevalence cohort allowed us to identify the prevalence of BMS and compare demographic and clinical characteristics of BMS according to the 3 most commonly used classification criteria $[2,3,18]$, as well as to identify prognostic factors associated with the disease course of BMS. Based on the classification criteria used, the overall

percentage of patients with BMS at enrollment was $19.8 \%$ to $33.3 \%$ at baseline, which is in accordance with previous studies $[2,10]$.

Our data suggest that patients with SPMS, PRMS, and PPMS disease course are less likely to remain benign over time than those with RRMS disease course, which is consistent with results previously reported in the literature $[10,18,24]$. Both baseline and follow-up data support the recommendations of others that the term "benign" MS should be reserved for patients with RRMS disease course [2, 3, 7, 8, 18, 22]. In cross-sectional baseline analyses, several demographic, clinical, and treatment factors increased the likelihood of being classified as benign. However, in exploratory Cox model analyses of benign patients over time, use of DMT and longer DD were the only significant prognostic factors for continued BMS. The data surrounding the use of DMTs suggest that there are benefits of early preventive treatment even in the absence of signs of physical disability. However, while follow-up analyses were limited to patients with baseline EDSS $\leq 2.0$ after 10 or more years of disease duration, correlation between EDSS score and DMT use was not directly studied herein. Nevertheless, these novel findings support the consensus statements issued by the NMSS in 2008 and the Consortium of Multiple sclerosis Centers (CMSC) in 2014 regarding early initiation of DMTs upon definitive MS diagnosis for patients and continuing treatment in those who are appropriate candidates for these agents [11, 12]. Use of DMTs early in the MS disease course has been supported by pharmacoeconomics studies that indicate early vs. delayed treatment with IFN- $\beta$, in particular, may be more cost-effective in the long term, as reduction of relapses, hospitalizations, and indirect costs, and gains in quality of life, and appear to outweigh the costs of DMTs $[14,15,25]$.

Our findings should be considered also in context of the study limitations. The diagnosis of MS at baseline and at follow-up was not based on McDonald but on Poser criteria [21, 26, 27]. Length of follow-up included in the follow-up analyses, was not the same for each patient but varied from 1 to 8 years. With the exception of 2 patients who were exposed to DMTs in clinical trials during the follow-up period, study patients were primarily treated with first-generation DMTs. Furthermore, this was a historical study, and available data did not allow us to examine every potential prognostic factor for BMS, including MRI. For example, because data on specific symptom at onset were not available for the cohort studied (i.e. optic neuritis, sensory symptoms, and longer time intervals from onset to the second attack), some factors previously shown to be prognostic of BMS were not examined herein $[10,18,24]$. Another limitation is the missing data on cognitive status, as EDSS used within this 
period did not include the cognitive functional score. Similarly, additional factors may be prognostic for patient loss to follow-up, and we may not have identified all possible causes of missing follow-up data. Also, while this was an exploratory study and we evaluated factors that predicted loss to follow-up, it is still possible that bias may have been introduced due to loss to follow-up. Our finding that less than $1 \%$ of progressive patients in the study cohort met criteria for BMS at baseline supports the notion that that progressive patients should not be classified as benign, regardless of whether they meet EDSS and DD criteria for benign classification.

Nevertheless, this study has several strengths, including the large, NYS-based, centralized dataset of patients with MS that includes almost 400 African-American patients, some of whom had BMS; a comparison of the prevalence of BMS according to 3 different definitions of benign status; and the availability of treatment data that allowed us to explore the potential effect of DMT on likelihood of continued benign status.

The results in the African-American subset of patients in our cohort are consistent with previous findings from our group and others that African-American patients with MS are likely to experience more rapid progression of disease than Caucasians [20, 28, 29]. However, while they were less likely to be classified as benign at baseline, African-American patients with BMS were just as likely to remain benign over time as Caucasians after controlling for other significant predictors of maintaining benign status. It is unlikely that this finding is the result of differential loss to follow-up or differing follow-up times because African-American race was not associated with loss to follow-up, and median follow-up years for AfricanAmericans with BMS was not significantly different from that of other BMS patients (4.5 vs 4.0 years).

On the basis of our historical cohort study findings and those from various studies reported in the literature, we support the recommendation of Pittock and others to narrow the definition of BMS to an EDSS $\leq 2$ after a minimum of 10 years' DD, at least until more reliable predictors of BMS (i.e. genetic, biologic, or imaging biomarkers) have been fully developed and validated $[2,8,22]$. Many patients retrospectively classified as having BMS may ultimately transition to a non-BMS, progressive form of MS, even using these more conservative criteria $[4,5,16,19,30]$.

\section{Conclusions}

Taken in context with previously reported benefits of the early initiation of DMT for patients diagnosed with MS $[4,9,16,25]$, our findings suggest that early initiation and continued treatment with DMT may increase the likelihood of maintaining BMS classification over the course of patients' lives, thereby ameliorating disability progression, and are worth further consideration and evaluation.

\section{Additional file}

Additional file 1: Figure S1. Study cohort diagram. Data from New York State Multiple Sclerosis Consortium. (TIF 377 kb)

\section{Abbreviations}

BMS, Benign MS; CDMS, Clinically definite multiple sclerosis; CMSC, Consortium of Multiple sclerosis Centers; DD, Disease duration; DMTs, Disease modifying therapies; EDSS, Expanded Disability Status Scale; FDA, Food and Drug Administration; HRs, Hazard ratios; IFN- $\beta$, Interferon beta-1; IRBs, Institutional Review Boards; LR, Logistic regression; MS, Multiple sclerosis; NMSS, National Multiple Sclerosis Society; NYS, New York State; NYSMSC, New York State MS Consortium; PPMS, Primary-progressive multiple sclerosis; PRMS, Progressiverelapsing multiple sclerosis; RRMS, Relapsing-remitting multiple sclerosis; SPMS, Secondary-progressive multiple sclerosis; SPSS, Statistical Package for Social Sciences; UDSMR, Centralized data management center

\section{Acknowledgements}

We would like to acknowledge the contributions of Drs. Steven Schwid, Carl Granger, Cornelia Mihai, Christopher Christodoulou, Neeta Garg, Kara Patrick, and Deepon P. Singh. Biogen provided funding for editorial support in the development of this paper, and Ray Hunziker (ProEd Communications, Inc.) copyedited and styled the manuscript per journal requirements. Biogen reviewed and provided feedback on the paper to the authors. The authors had full editorial control of the paper, and provided their final approval of all content.

Funding

Biogen provided support for publication of this report.

Availability of data and materials

Availability of data and materials will not be made available in order to protect the participant's identity.

\section{Authors' contributions}

RZ, BW-G, and DLC substantially contributed to the concept and design of the study. RZ and DLC performed the statistical analysis. RZ and DLC drafted the first version of the article. BW-G, LK, AEM, NL, PKC, ADG, BJ, ML, JH, MG, $D H S, B R A, B E T, A B P$, and FM were responsible for recruitment and accrual of data for the registry and revised the article critically for important intellectual content. All authors had access to the data. All authors read and approved the final manuscript.

\section{Authors' information}

Not applicable.

\section{Competing interests}

Dr. Zivadinov received personal compensation from Teva Pharmaceuticals, Biogen, EMD Serono, Novartis, Claret Medical, and Sanofi-Genzyme for presentation and consultant fees, and has received financial support for research activities from Biogen, Teva Pharmaceuticals, EMD Serono, Novartis, Claret Medical, Greatbatch, and Sanofi-Genzyme.

Dr. Weinstock-Guttman has participated in speaker's bureaus and served as a consultant for Biogen, Teva Neurosciences, EMD Serono, Pfizer, Novartis, Genzyme, and Acorda. She also has received grant/research support from the agencies listed above as well as Questcor. No other industry financial relationships exist

Dr. Cookfair has received compensation from Buffalo Neuroimaging and Analysis Center (BNAC) as part of a contractual agreement to conduct statistical analyses on this and other projects for BNAC. No other financial/ competing relationships to declare.

Dr. Krupp reports no disclosures.

Dr. Miller is a consultant for Acorda, Biogen, CVS Caremark, EMD Serono, Genzyme/Sanofi, GlaxoSmithKline, Novartis, Questcor, Roche, and Teva and has received research support from Acorda, Biogen, Genentech, Novartis, Questcor, and Roche. 
Dr. Lava received research support from Biogen, Sanofi-Genzyme, and Roche. Dr. Coyle has served as a consultant for AbbVie, Accordant, Acorda Therapeutics, Bayer, Biogen, Genentech/Roche, Genzyme/Sanofi, Novartis, EMD Serono, and Teva. Dr. Coyle's employer, Stony Brook University Medical Center, has received research support for conducting clinical trials from Actelion, Novartis, and Opexa.

Dr. Goodman received personal compensation for consulting from the following commercial entities: Acorda Therapeutics, Biogen, EMD-Serono, Genzyme-sanofi, GW Pharma, Novartis, Purdue, Teva, and Vaccinex. Dr. Goodman's employer, the University of Rochester, received research support for conducting clinical trials from the following commercial entities: Acorda Therapeutics, Avanir, Biogen, EMD-Serono, Genzyme-sanofi, Novartis, Ono, Roche, Sun Pharma, Teva. Dr. Jubelt has received grant support from Sanofi-Aventis, Genzyme, Novartis, NY Stem Cell Program, NINDS/MediciNova, Inc., and Biogen is a consultant for Teva Neuroscience and Genzyme, and holds patents/royalty for UpToDate. He is also a lecturer for CMEducation Resources LLC, Prime Education, Inc., National Multiple Sclerosis Society, and Multiple Sclerosis Resources of Central New York. Dr. Lenihan reports no disclosures.

Dr. Herbert reports no disclosures.

Dr. Gottesman has received consulting fees from Biogen, Teva, and Genzyme. Dr. Snyder reports no disclosures.

Dr. Apatoff has received consulting fees from Biogen, Teva, and Genzyme. Dr. Teter has received research grant support from Biogen, Teva Neurosciences, EMD Serono, Avanir, Genzyme, and Novartis. Dr. Perel reports no disclosures.

Dr. Munschauer is a former employee of Biogen and a current employee of EMD Serono.

\section{Consent for publication}

All authors consented the right for publication.

\section{Ethics approval and consent to participate}

The study was approved by the relevant Institutional Review Boards (IRBs) of the participating centers and written informed consent was obtained by all study participants prior to enrollment in the NYSMSC registry. The IRB renewals were obtained annually on an ongoing basis throughout the study.

\section{Author details}

'Buffalo Neuroimaging Analysis Center, Department of Neurology, School of Medicine and Biomedical Sciences, University at Buffalo, Buffalo, NY, USA. ${ }^{2}$ Department of Neurology, School of Medicine and Biomedical Sciences, University at Buffalo, 100 High Street, Buffalo, NY 14203, USA. ${ }^{3}$ Department of Neurology, Stony Brook University Medical Center, Stony Brook, NY, USA. ${ }^{4}$ The Corinne Goldsmith Dickinson Center of Multiple Sclerosis, Mount Sinai School of Medicine, New York, NY, USA. ${ }^{5}$ Department of Neurology, Albany Medical School, Multiple Sclerosis Center, Albany, NY, USA. ${ }^{6}$ Department of Neurology, University of Rochester Medical Center, Rochester, NY, USA. ${ }^{7}$ Department of Neurology, SUNY Upstate Medical University, Syracuse, NY, USA. ${ }^{8}$ Adirondack Neurology Associates, Glens Falls, NY, USA. ${ }^{9}$ Department of Neurology, NYU School of Medicine, New York, NY, USA. ${ }^{10}$ Department of Neuroscience, Winthrop University Hospital, Mineola, NY, USA. ${ }^{11}$ Department of Neurology, Weill Medical College of Cornell University, New York, NY, USA. ${ }^{12}$ Department of Neurology, Albert Einstein College of Medicine, New York, NY, USA. ${ }^{13}$ Multiple Sclerosis Center, New York, NY, USA. ${ }^{14}$ Alpha Neurology, Staten Island, NY, USA.

Received: 7 January 2016 Accepted: 15 June 2016

\section{Published online: 15 July 2016}

\section{References}

1. Compston A, Coles A. Multiple sclerosis. Lancet. 2002;359:1221-31. doi:10.1016/S0140-6736(02)08220-X

2. Correale J, Ysrraelit MC, Fiol MP. Benign multiple sclerosis: does it exist? Curr Neurol Neurosci Rep. 2012;12:601-9. doi:10.1007/s11910-012-0292-5.

3. Glad SB, Aarseth JH, Nyland $H$, et al. Benign multiple sclerosis: a need for a consensus. Acta Neurol Scand Suppl 2010:44-50. doi:10.1111/j. 1600-0404.2010.01375.x

4. Gold R, Wolinsky JS, Amato MP, et al. Evolving expectations around early management of multiple sclerosis. Ther Adv Neurol Disord. 2010;3:351-67.
5. Sayao AL, Bueno AM, Devonshire $V$, et al. The psychosocial and cognitive impact of longstanding 'benign' multiple sclerosis. Mult Scler. 2011;17:1375-83. doi:10.1177/1352458511410343.

6. Lublin FD, Reingold SC. Defining the clinical course of multiple sclerosis: results of an international survey. National Multiple Sclerosis Society (USA) Advisory Committee on Clinical Trials of New Agents in Multiple Sclerosis. Neurology. 1996;46:907-11.

7. Lublin FD, Reingold SC, Cohen JA, et al. Defining the clinical course of multiple sclerosis: the 2013 revisions. Neurology. 2014;83:278-86. doi:10.1212/WNL.0000000000000560.

8. Leray $\mathrm{E}$, Coustans $\mathrm{M}$, Le Page $\mathrm{E}$, et al. 'Clinically definite benign multiple sclerosis', an unwarranted conceptual hodgepodge: evidence from a 30-year observational study. Mult Scler. 2013;19:458-65.

9. Ramsaransing G, Maurits N, Zwanikken C, et al. Early prediction of a benign course of multiple sclerosis on clinical grounds: a systematic review. Mult Scler. 2001;7:345-7.

10. Ramsaransing GS, De Keyser J. Benign course in multiple sclerosis: a review. Acta Neurol Scand. 2006;113:359-69. doi:10.1111/j.1600-0404.2006.00637.x.

11. Multiple Sclerosis Coalition. The use of disease-modifying therapies in multiple sclerosis: principles and current evidence. A consensus paper by the Multiple Sclerosis Coalition. Available at: http://www.nationalmssociety. org/getmedia/5ca284d3-fc7c-4ba5-b005-ab537d495c3c/DMT_Consensus_ MS_Coalition_color. 2015. Accessed July 10, 2015.

12. National Multiple Sclerosis Society. Disease management consensus statement. Available at: http://www.nationalmssociety.org/aboutmultiple-sclerosis/what-we-know-about-ms/treatments/index.aspx. 2008 Accessed July 10, 2015

13. Tornatore C, Phillips JT, Khan O, et al. Practice patterns of US neurologists in patients with CIS, RRMS, or RIS: a consensus study. Neurol Clin Pract. 2012:2:48-57. doi:10.1212/CPJ.0b013e31824cb09b.

14. Trojano M, Paolicelli D, Lepore $\mathrm{V}$, et al. Italian Multiple Sclerosis Database Network. Neurol Sci. 2006:27 suppl 5:S358-S61. doi:10.1007/ s10072-006-0694-8.

15. Trojano M, Russo P, Fuiani A, et al. The Italian Multiple Sclerosis Database Network (MSDN): the risk of worsening according to IFNbeta exposure in multiple sclerosis. Mult Scler. 2006;12:578-85.

16. Jacobs LD, Wende KE, Brownscheidle CM, et al. A profile of multiple sclerosis: the New York State Multiple Sclerosis Consortium. Mult Scler. 1999:5:369-76.

17. New York State Multiple Sclerosis Consortium. Demographic and clinical data to promote MS research and enhance patient care. Available at: http://www.nysmsc.org/docs/brochure.pdf. Accessed July 10, 2015.

18. Correale J, Peirano I, Romano L. Benign multiple sclerosis: a new definition of this entity is needed. Mult Scler. 2012;18:210-8. doi:10.1177/1352458511419702.

19. Hawkins SA, McDonnell GV. Benign multiple sclerosis? Clinical course, long term follow up, and assessment of prognostic factors. J Neurol Neurosurg Psychiatry. 1999;67:148-52

20. Weinstock-Guttman B, Jacobs LD, Brownscheidle CM, et al. Multiple sclerosis characteristics in African American patients in the New York State Multiple Sclerosis Consortium. Mult Scler. 2003:9:293-8.

21. Poser CM, Paty DW, Scheinberg L, et al. New diagnostic criteria for multiple sclerosis: guidelines for research protocols. Ann Neurol. 1983;13:227-31. doi:10.1002/ana.410130302

22. Pittock SJ, McClelland RL, Mayr WT, et al. Clinical implications of benign multiple sclerosis: a 20-year population-based follow-up study. Ann Neurol. 2004;56:303-6. doi:10.1002/ana.20197.

23. Freedman MS, Selchen D, Arnold DL, et al. Treatment optimization in MS: Canadian MS Working Group updated recommendations. Can J Neurol Sci. 2013:40:307-23.

24. Glad S, Nyland H, Myhr KM. Benign multiple sclerosis. Acta Neurol Scand Suppl. 2006;183:55-7. doi:10.1111/j.1600-0404.2006.00617.x.

25. Castrop F, Haslinger B, Hemmer B, et al. Review of the pharmacoeconomics of early treatment of multiple sclerosis using interferon beta. Neuropsychiatr Dis Treat. 2013;9:1339-49.

26. Polman $\mathrm{CH}$, Reingold SC, Banwell B, et al. Diagnostic criteria for multiple sclerosis: 2010 revisions to the McDonald criteria. Ann Neurol. 2011:69:292-302. doi:10.1002/ana.22366.

27. Polman $\mathrm{CH}$, Reingold SC, Edan G, et al. Diagnostic criteria for multiple sclerosis: 2005 revisions to the "McDonald Criteria". Ann Neurol. 2005:58: 840-6. doi:10.1002/ana.20703. 
28. Cree BA, Khan O, Bourdette D, et al. Clinical characteristics of African Americans vs Caucasian Americans with multiple sclerosis. Neurology. 2004;63:2039-45.

29. Kister I, Chamot E, Bacon JH, et al. Rapid disease course in African Americans with multiple sclerosis. Neurology. 2010;75:217-23. doi:10.1212/ WNL.0b013e3181e8e72a.

30. Sayao AL, Devonshire V, Tremlett H. Longitudinal follow-up of "benign" multiple sclerosis at 20 years. Neurology. 2007:68:496-500. doi:10.1212/01. wnl.0000253185.03943.66.

Submit your next manuscript to BioMed Central and we will help you at every step:

- We accept pre-submission inquiries

- Our selector tool helps you to find the most relevant journal

- We provide round the clock customer support

- Convenient online submission

- Thorough peer review

- Inclusion in PubMed and all major indexing services

- Maximum visibility for your research

Submit your manuscript at www.biomedcentral.com/submit 